\title{
Clinical Significance of Serum CA125, CA19-9, CA72-4, and Fibrinogen-to-Lymphocyte Ratio in Gastric Cancer With Peritoneal Dissemination
} Chao Huang, Zitao Liu, Li Xiao, Yongqiang Xia, Jun Huang, Hongliang Luo, Zhen Zong and
Zhengming Zhu*

Department of Gastrointestinal Surgery, The Second Affiliated Hospital of Nanchang University, Nanchang, China

Background: Relevant serum tumor markers have been indicated to be associated with peritoneal dissemination (PD) of gastric cancer (GC). Fibrinogen has been shown to play an important role in the systemic inflammatory response (SIR) and in tumor progression. However, the clinical significance of the fibrinogen-to-lymphocyte ratio (FLR) in GC with PD has not been studied.

OPEN ACCESS

Edited by:

Samuel J. Klempner, Massachusetts General Hospital Cancer Center, United States

Reviewed by: Johan Nicolay Wiig, Oslo University Hospital, Norway Jinping Liu,

Perelman School of Medicine, University of Pennsylvania United States

*Correspondence: Zhengming Zhu zzm8654@163.com

Specialty section:

This article was submitted to Gastrointestinal Cancers, a section of the journal Frontiers in Oncology

Received: 09 August 2019 Accepted: 17 October 2019 Published: 05 November 2019

Citation:

Huang C, Liu Z, Xiao L, Xia Y, Huang J, Luo H, Zong Z and Zhu Z (2019) Clinical Significance of Serum CA125, CA19-9, CA72-4, and Fibrinogen-to-Lymphocyte Ratio in Gastric Cancer With Peritoneal Dissemination. Front. Oncol. 9:1159.

doi: 10.3389/fonc.2019.01159
Methods: The clinical data of 391 patients with GC were collected, including 86 cases of PD. Then, 1:3 matching was performed by propensity score matching (PSM), and the clinical data of the matched 344 patients were analyzed by univariate and multivariate conditional logistic regression. Classification tree analysis was used to obtain the decision rules and a random forest algorithm to extract the important risk factors of PD in GC. A nomogram model for risk assessment of PD in GC was established by using the rms package of $\mathrm{R}$ software.

Results: Univariate analysis showed that the factors related to $P D$ in GC were: carbohydrate antigen (CA) 125 ( $P<0.0001)$, CA19-9 $(P<0.0001)$, CA72-4 $(P<$ 0.0001), FLR $(P<0.0001)$, neutrophil-to-lymphocyte ratio $(N L R)(P<0.0001)$, albuminto- lymphocyte ratio (ALR) $(P<0.0001)$, platelet-to-lymphocyte ratio (PLR) $(P=0.013)$, and carcinoembryonic antigen (CEA) $(P=0.031)$. Conditional logistic regression found that CA125 (OR: 1.046; $P<0.0001)$, CA19-9 (OR: 1.002; $P<0.0001)$, and FLR (OR: 1.266; $P=0.024$ ) were independent risk factors for $G C$ with $P D$. The accuracy, sensitivity, specificity, positive predictive value and negative predictive value of the decision rules for detecting PD of GC were 89.5, 77.4, 94.0, 82.8, and 91.8\%, respectively. According to the important variables identified by the classification tree and random forest algorithm, the risk assessment model of PD in GC was established. The accuracy, sensitivity, and specificity of the model were $91,89.5$, and $79.5 \%$, respectively.

Conclusion: CA125 > 17.3 U/ml, CA19-9 > 27.315 U/ml, and FLR > 2.555 were the risk factors for $\mathrm{GC}$ with $\mathrm{PD}$. The decision rules and nomogram model constructed by CA125, CA19-9, CA72-4, and FLR can correctly predict the risk of PD in GC.

Keywords: gastric cancer, peritoneal dissemination, risk factors, decision rules, risk assessment model 


\section{INTRODUCTION}

Gastric cancer (GC) is a malignant tumor with a high incidence ranking fifth among all cancers, but its mortality rate ranks third in the global cancer mortality rate (1). Peritoneal dissemination (PD) is a common distant metastasis mode of GC, and the most important factor leading to shortened survival of patients (2). Relevant guidelines $(3,4)$ clearly stipulate that GC with PD should not be treated with radical surgery and only with palliative treatment. Therefore, accurate prediction of $\mathrm{PD}$ in GC can not only avoid unnecessary laparotomy but also provide the opportunity for early comprehensive treatment such as chemotherapy. At present, computed tomography (CT) examination as a common means to determine the PD of GC has high accuracy and specificity, but the sensitivity is low; PETCT examination has difficulty in detecting nodules below $5 \mathrm{~mm}$, while the lesions of $\mathrm{PD}$ are usually small, so there is a high rate of missed diagnoses (5-7). Although laparoscopic exploration is highly accurate, it is an invasive procedure. Therefore, it is of great significance to identify a non-invasive, highly sensitive and simple prediction method. In recent years, studies have shown that serum tumor markers, especially CA125, have important clinical value in the diagnosis of PD in GC, but the sensitivity of each single indicator was low (8-10). In addition, relevant studies $(11,12)$ have shown that systemic inflammatory response (SIR) plays an important role in the occurrence, progression and metastasis of tumors. Fibrinogen, a $340-\mathrm{kDa}$ liver glycoprotein, has been proven to play an important role in SIR and tumor progression (13-18). Some studies have also shown that elevated fibrinogen levels are associated with tumor progression and metastasis in advanced gastric cancer (19-21). However, the clinical significance of fibrinogen-to-lymphocyte ratio (FLR) in GC with PD has not been studied. Therefore, the purpose of this study was to investigate the clinical significance of CA125, CA19-9, CA72-4, and FLR in GC with PD.

\section{METHODS}

This was a retrospective study and performed according to the guidelines of the Helsinki Declaration. The study was approved by the Ethical Committee of the Second Affiliated Hospital of Nanchang University and written informed consent was obtained from all patients.

\section{Study Patients}

The clinical data of 391 patients with GC were collected, including 86 cases of PD. PD refers to the infiltration of GC tissue into the serosal, and the tumor cells are shed and planted on the peritoneum and organ serosal to form metastatic nodules. The presence of such nodules on CT examination or during surgery was defined as PD group. The absence of such nodules during surgery was defined as without PD group. A total of 391 patients with GC were matched 1:3 according to age, sex, and body mass index (BMI). A total of 344 patients were successfully matched. There were 86 cases in the PD group, including 60 men and 26 women. Their median age was 64 years and their median BMI was $21.38 \mathrm{~kg} / \mathrm{m}^{2}$. There were 258 cases in the without PD group
TABLE 1 | General characteristics of patients with gastric cancer and comparison of the relevant factors between the two groups $(n=344)$.

\begin{tabular}{lccc}
\hline Factor & $\begin{array}{l}\text { PD } \\
(\boldsymbol{n}=\mathbf{8 6})\end{array}$ & $\begin{array}{c}\text { Without PD } \\
(\boldsymbol{n}=\mathbf{2 5 8})\end{array}$ & $\boldsymbol{P}$ value \\
\hline Age (years) & $64(52,71)$ & $64(55,69)$ & 0.900 \\
Sex (n) & & & 0.773 \\
Male & 60 & 184 & \\
Female & 26 & 74 & \\
BMl (kg/m²) & $21.38(18.86,22.55)$ & $21.51(19.48,23.46)$ & 0.600 \\
Indicators & & & \\
NLR & $3.58(2.11,5.58)$ & $2.44(1.76,3.60)$ & $<0.0001$ \\
PLR & $188.88(139.74,266.67)$ & $158.86(117.68,211.48)$ & 0.013 \\
ALR & $32.13(24.73,41.86)$ & $26.15(22.14,34.84)$ & $<0.0001$ \\
FLR & $2.91(2.16,4.22)$ & $2.07(1.52,2.97)$ & $<0.0001$ \\
Hb (g/L) & $119.5(98.50,133.25)$ & $124.5(104.75,139.25)$ & 0.560 \\
CEA (ng/ml) & $5.60(2.89,17.12)$ & $3.08(1.86,5.98)$ & 0.031 \\
CA19-9 (U/ml) & $33.60(13.49,593.18)$ & $13.73(7.69,24.04)$ & $<0.0001$ \\
CA125 (U/ml) & $41.65(19.95,98.23)$ & $8.35(5.98,13.85)$ & $<0.0001$ \\
CA72-4 (IU/ml) & $9.86(2.18,23.40)$ & $1.84(1.20,7.03)$ & $<0.0001$ \\
\hline
\end{tabular}

$P D$, peritoneal dissemination; $B M I$, body mass index; NLR, neutrophil-to-lymphocyte ratio; $H b$, hemoglobin; PLR, platelet-to-lymphocyte ratio; ALR, albumin-to- lymphocyte ratio; $F L R$, fibrinogen-to-lymphocyte ratio.

(diagnosed by laparotomy), including 184 men and 74 women. Their median age was 64 years and their median BMI was 21.51 $\mathrm{kg} / \mathrm{m}^{2}$. There were no statistically significant differences in age $(P$ $=0.900)$, sex $(P=0.773)$, BMI $(P=0.600)$, or hemoglobin $(\mathrm{Hb})$ $(P=0.560)$ between the two groups (Table $\mathbf{1})$.

\section{Inclusion and Exclusion Criteria}

The inclusion criteria were as follows: (1) Patients with GC were diagnosed by surgery or CT examination; (2) Neoadjuvant radiotherapy or chemotherapy was not administered before surgery; (3) Patients signed informed consent forms; (4) It was approved by the ethics committee of our hospital. The exclusion criteria were as follows: (1) Received surgical treatment within 2 months of enrollment; (2) A history of blood transfusion, bleeding, hemostasis, anticoagulant drugs, or thrombosis; (3) Patient underwent splenectomy; (4) Patients with pregnancy, diabetes, cirrhosis, nephrotic syndrome, acute infection, other distant organ metastasis, or incomplete data were excluded.

\section{Statistical Analysis}

We used the single-sample k-s test to test the normality of the data. If the quantitative data had a normal distribution, they are described as the mean \pm standard deviation; otherwise, they are described as the median and interquartile range. Categorical variables are expressed as rate with $95 \%$ confidence intervals (CI). Univariate and multivariate logistic regression were used to analyze the indicators of the matched patients. Classification tree analysis was used to obtain the decision rules and a random forest algorithm to extract the important risk factors of PD in GC. A classification tree is a non-linear discriminant method that uses a set of independent variables to gradually decompose a sample into smaller subgroups. This procedure selects the 
TABLE 2 | Risk factors of PD in GC for multivariate conditional logistic regression analysis.

\begin{tabular}{lccccc}
\hline Risk factors & B & SE & Wals & $\boldsymbol{P}$ value & OR (95\% CI) \\
\hline CA125 & 0.045 & 0.009 & 26.817 & $<0.0001$ & $1.046(1.028,1.064)$ \\
CA19-9 & 0.002 & 0.000 & 13.612 & $<0.0001$ & $1.002(1.001,1.003)$ \\
FLR & 0.236 & 0.105 & 5.063 & 0.024 & $1.266(1.031,1.555)$ \\
\hline
\end{tabular}

independent variable that has the strongest association with the dependent variable (22). The decision rules provide specific information about risk factors based on rule induction. The direction of individual movement is determined by the answers to the questions on each branch until reaching the end of a terminal node (ellipse) (23). A classification tree model was built by randomly extracting two-thirds of all data (24). The important indicators were ranked by the mean decrease Gini (MDG) involved in the random forest algorithm. The MDG provides a method to quantify which indicator contributes the most to the classification accuracy (25). The threshold of important risk factors was determined by the receiver operating characteristic curve (ROC curve). The optimal cut-off value is commonly used in the "Youden index," i.e., sensitivity-(1-specificity), where the maximum value of the index is the optimal threshold. The $\mathrm{R}$ software rms package was used to construct the nomogram model of risk assessment for PD in GC, and points of various indicators were obtained (26). The points corresponding to the indicators were added to obtain the total points; the higher the total points, the higher the risk of PD of GC. The ROC curve was used to evaluate the accuracy, sensitivity and specificity of the model in predicting PD in GC. A two-sided $P<0.05$ was considered statistically significant. Data were analyzed using SPSS 22.0 for Windows (SPSS Inc., Chicago, IL, USA) and R (version x64 3.5.1, http://www.r-project.org).

\section{RESULTS}

\section{Univariate and Multivariate Analysis}

Univariate analysis showed that the factors related to PD in GC were as follows: CA125 $(P<0.0001)$, CA199 $(P<0.0001)$, CA72$4(P<0.0001)$, FLR $(P<0.0001)$, NLR $(P<0.0001)$, ALR $(P$ $<0.0001)$, PLR $(P=0.013)$, and CEA $(P=0.031)$, and the differences between the two groups were statistically significant (Table 1). Multivariate analysis found that CA125 (OR: 1.046; $P<0.0001$ ), CA19-9 (OR: 1.002; $P<0.0001$ ), and FLR (OR: 1.266; $P=0.024)$ were independent risk factors for GC with PD (Table 2). The accuracy, sensitivity and specificity of predicting PD in GC were 89.3, 82.6, and 88.4\%, respectively (Figure 1).

\section{Classification Tree Analysis to Obtain Decision Rules Affecting PD in GC}

Eight variables with statistically significant differences were analyzed by using a classification tree to obtain decision rules affecting PD in GC (Figure 2). Four variables were selected through the classification tree program. They were CA125, CA19-9, CA72-4, and FLR. CA125 was the most important

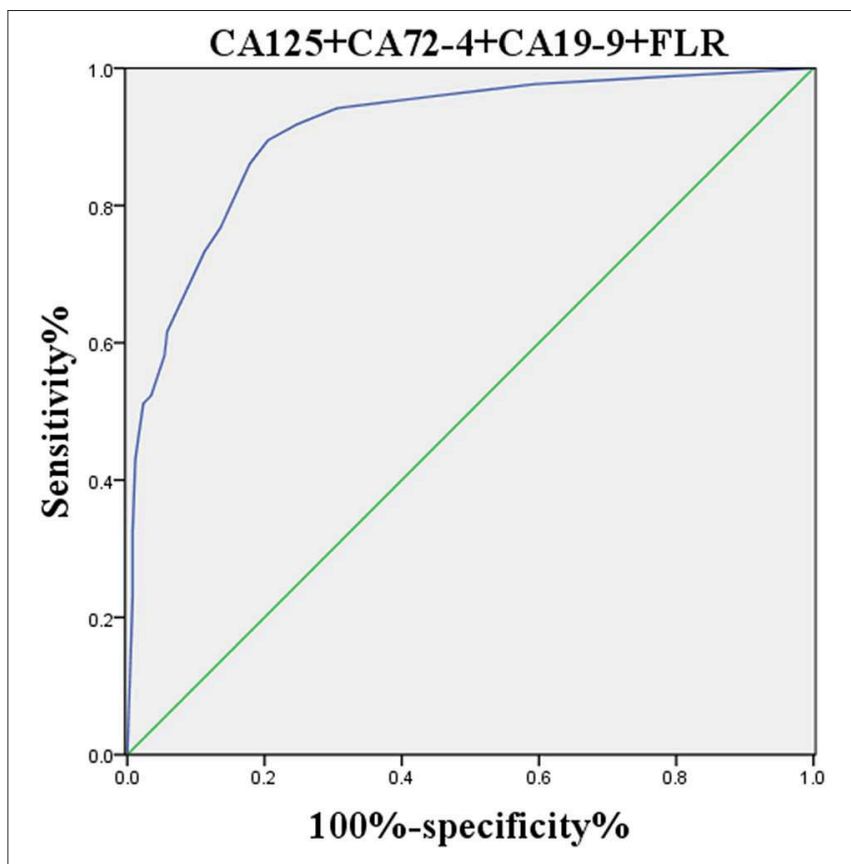

FIGURE 1 | ROC curve of independent risk factors of PD in GC.

determining factor because it was the first-level split of the two initial branches of the decision tree. CA72-4 was the most important determining factor in the second-level split. The accuracy, sensitivity, specificity, positive predictive value and negative predictive value of the decision rules for detecting PD of GC were $89.5,77.4,94.0,82.8$, and $91.8 \%$, respectively, indicating that the decision rules had high accuracy and specificity.

\section{Random Forest Algorithm to Extract Important Risk Factors for PD of GC}

All variables were analyzed using a random forest algorithm to obtain the importance ranking of the variables (Figure 3). The larger the mean decrease of Gini, the more important the indicator was. The first was CA125, followed by CA72-4, CA19-9, and FLR.

\section{ROC Curve of Important Risk Factors}

Figure 4 shows the correlation between CA125, CA72-4, CA199, and FLR with PD in GC. The optimal cut-off values of CA125, CA72-4, CA19-9, and FLR were $17.3 \mathrm{U} / \mathrm{ml}, 7.25 \mathrm{IU} / \mathrm{ml}$, $27.315 \mathrm{U} / \mathrm{ml}$ and 2.555, respectively, according to the ROC curve evaluation. The area under the curve (AUC) of CA125 was 0.820 , the $95 \%$ CI was $0.764-0.876$, the sensitivity was $79.1 \%$, and the specificity was $84.9 \%$. The AUC of CA72- 4 was 0.717 , the $95 \%$ CI was $0.649-0.785$, the sensitivity was $57 \%$, and the specificity was $86.4 \%$. The AUC of CA19-9 was 0.684 , the $95 \%$ CI was $0.615-$ 0.753 , the sensitivity was $57 \%$, and the specificity was $79.8 \%$. The AUC of FLR was 0.653 , the $95 \%$ CI was $0.586-0.720$, the sensitivity was $65.1 \%$, and the specificity was $65.5 \%$ (Table 3 ). These data indicate that the accuracy and sensitivity of individual indicators to determine PD were relatively low. 


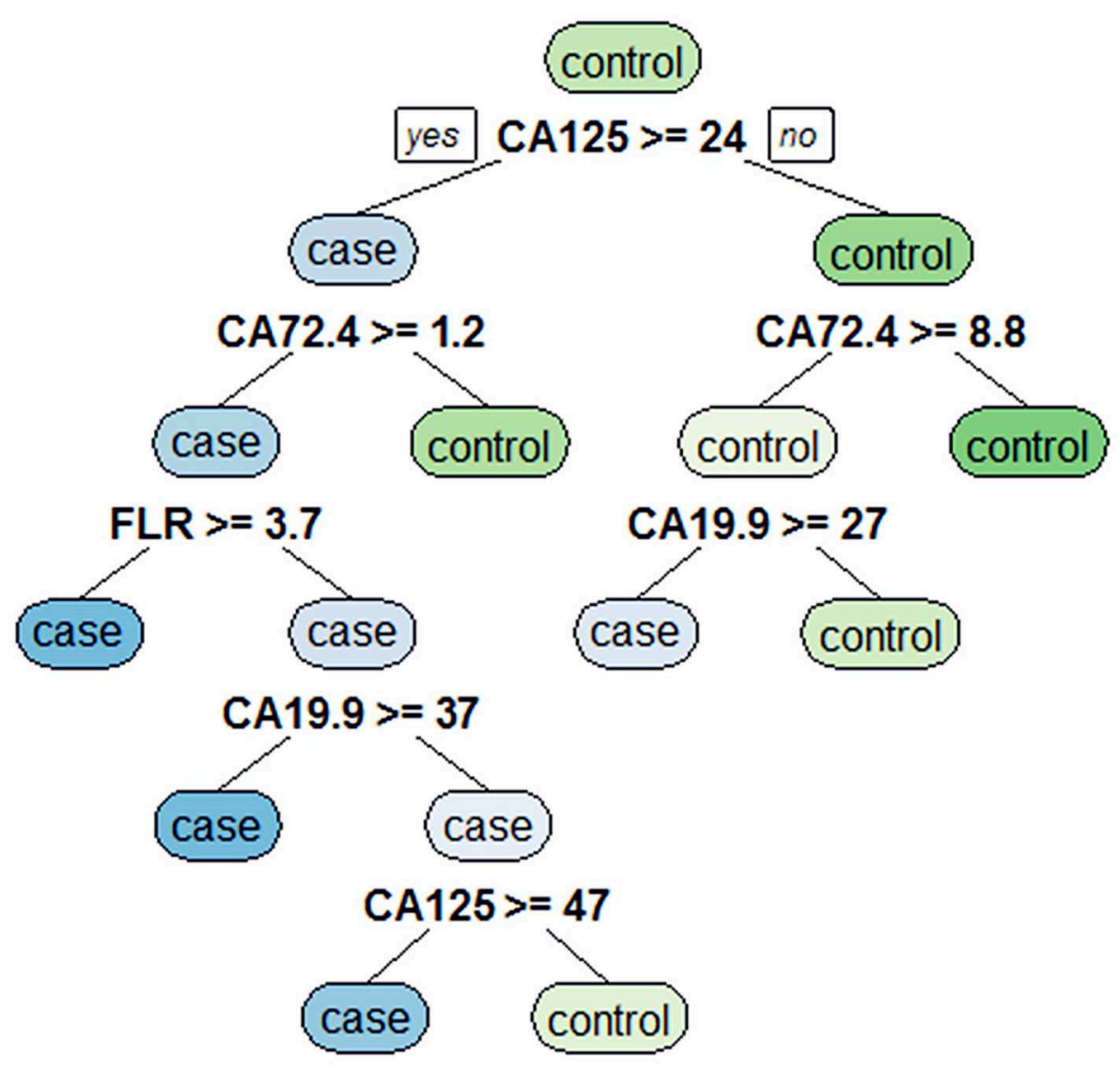

FIGURE 2 | Classification tree for detecting undiagnosed PD in GC.

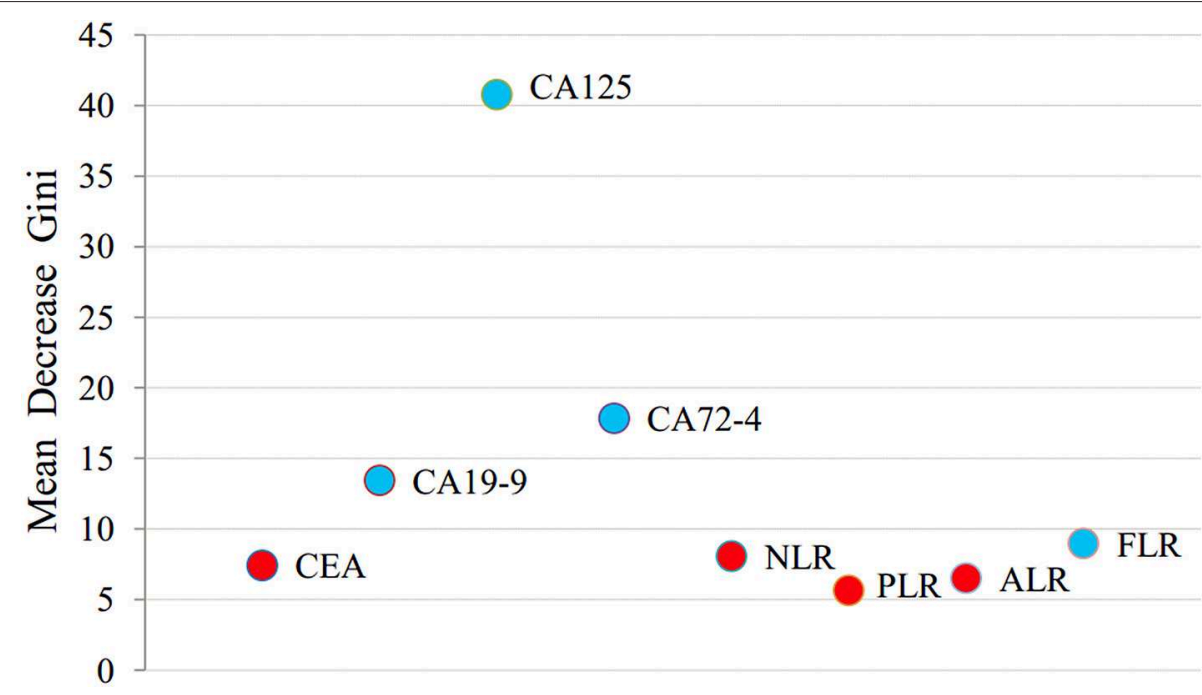

FIGURE 3 | Random forest algorithm for ranking of the importance of all variables. 


\section{Nomogram Model for Risk Assessment of GC With PD}

The logistic regression model of CA125, CA72-4, CA19-9, and FLR was constructed by the $\mathrm{R}$ language rms package, and the C statistic of its evaluation was 0.910 , demonstrating that the prediction model had high accuracy. Then, the plotting function was constructed, and the nomogram was plotted (Figure 5). A score of CA125>17.3 U/ml was 100 points, while a score of CA125 $\leq 17.3 \mathrm{U} / \mathrm{ml}$ was 0 points; a score of CA72-4 $>7.24$ $\mathrm{IU} / \mathrm{ml}$ was 68 points, while a score of CA72-4 $\leq 7.24 \mathrm{IU} / \mathrm{ml}$ was 0 points; a score of CA19-9 > 27.315 U/ml was 41 points, while a score of CA19-9 $\leq 27.315 \mathrm{U} / \mathrm{ml}$ was 0 points; a score of FLR $>2.555$ was 27 points, while a score of PAR $\leq 2.555$ was 0 points. The total score was 236 points, suggesting that the probability of PD in GC was 91-95\%. The risk of GC with PD can be predicted based on the total points (Table 4). The area under the ROC curve of the combined factors was 0.910 , the $95 \% \mathrm{CI}$ was $0.873-0.946$, the sensitivity was $89.5 \%$, and the specificity was $79.5 \%$, indicating that the prediction model had high accuracy and sensitivity (Figure 6).

\section{DISCUSSION}

At present, CT examination is a common method to diagnose PD of GC. Although it has high accuracy and specificity, its sensitivity is low. It is also difficult to find nodules $<5 \mathrm{~mm}$

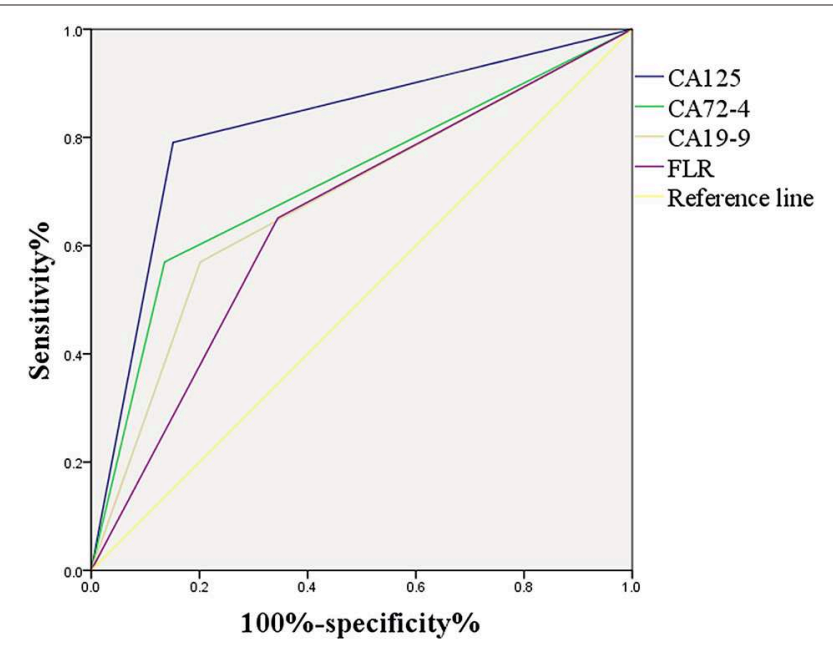

FIGURE 4 | ROC curve of the important risk factors. in PET/CT examination, and the lesions of $\mathrm{PD}$ are usually small, so there is a high rate of missed diagnosis (5-7). Kim et al. (5) found that the sensitivity of CT diagnosis of GC with PD was $28.3-50.9 \%$, and the specificity was $96.2-98.9 \%$ through retrospective analysis. Kayaalp et al. (27) analyzed 118 patients with GC using preoperative CT staging, indicating that the sensitivity, specificity, and accuracy of CT in diagnosing PD of GC were 13, 99, and $82 \%$, respectively. In addition, Yajima et al. (28) analyzed 293 patients with advanced gastric cancer, showing that the sensitivity, specificity, and accuracy of CT diagnosis of PD were 50.6, 96.8, and 85.0\%, respectively. Laparoscopic exploration is highly accurate, but it is an invasive procedure. In addition, cytological examination of peritoneal lavage during laparotomy can predict $\mathrm{PD}(29,30)$. In this study, 47 out of 86 (54.6\%) of patients with negative CT were identified to have PD by laparotomy. SIR plays an important role in the occurrence, progression and metastasis of tumors $(11,12)$. Chronic inflammation produces a microenvironment that facilitates tumor growth and metastatic spread of tumor cells $(31,32)$. Lymphocytes are major anticancer factors. As an important part of tumor-specific immune responses, they play a specific role in killing tumor cells by inducing cytotoxic cell death and cytokine production to mediate host immune responses, thereby inhibiting tumor cell proliferation $(12,33)$. Eo et al. (34) found that preoperative lymphocytosis was an independent risk factor for the prognosis of GC, and the overall survival and disease-free survival rates of patients with preoperative lymphocytosis were lower. In addition, Arigami et al. (35) showed that the density of the cluster of differentiation (CD) $3+$ tumorinfiltrating lymphocytes in patients with GC decreases during tumor progression.

Fibrinogen plays an important role in blood coagulation, cellcell adhesion, and the inflammatory response (19). Meanwhile, the coagulation cascade plays an important role in tumor progression and metastasis (36). Fibrinogen, as an important component of the coagulation cascade, has been proven to be an important regulator of SIR and tumor progression (1318). Fibrinogen provides a stable framework for the tumor extracellular matrix, thereby promoting tumor angiogenesis and enhancing adhesion, migration, and invasion of tumor cells (37); in addition, fibrinogen, as an acute phase protein, may induce tumor cell proliferation and invasion by mediating the initial adhesion of leukocytes to endothelial cells, thus promoting the release of pro-inflammatory cytokines (38). Previous studies (39) have reported that platelets and fibrinogen interact to protect tumor cells from the phagocytosis of natural killer cells. It is well-known that elevated fibrinogen is a predictor

TABLE 3 | Relevant results of important risk factors.

\begin{tabular}{|c|c|c|c|c|c|c|c|}
\hline Factors & AUC & SE & $P$ value & $95 \% \mathrm{Cl}$ & Sensitivity (\%) & Specificity (\%) & Cut-off value \\
\hline CA125 & 0.820 & 0.029 & $<0.0001$ & $(0.764,0.876)$ & 79.1 & 84.9 & 17.3 \\
\hline CA72-4 & 0.717 & 0.035 & $<0.0001$ & $(0.649,0.785)$ & 57 & 86.4 & 7.25 \\
\hline CA19-9 & 0.684 & 0.035 & $<0.0001$ & $(0.615,0.753)$ & 57 & 79.8 & 27.315 \\
\hline FLR & 0.653 & 0.034 & $<0.0001$ & $(0.586,0.720)$ & 65.1 & 65.5 & 2.555 \\
\hline
\end{tabular}




\begin{tabular}{l}
\hline CA72.4 \\
FLR \\
Total Points \\
Linear Predictor \\
Risk of PD \\
FIGURE 5 | Nomogram of the logistic regression model.
\end{tabular}

TABLE 4 | Relationship between total points and risk of PD in GC.

\begin{tabular}{lc}
\hline Total points & Risk of PD (\%) \\
\hline$<30$ & $<5$ \\
$30-56$ & $5-10$ \\
$57-85$ & $11-20$ \\
$86-104$ & $21-30$ \\
$105-120$ & $31-40$ \\
$121-134$ & $41-50$ \\
$135-149$ & $51-60$ \\
$150-164$ & $61-70$ \\
$165-183$ & $71-80$ \\
$184-212$ & $81-90$ \\
$213-239$ & $91-95$ \\
$>239$ & $>95$ \\
\hline
\end{tabular}

of cardiovascular events and mortality from chronic kidney disease (40). In addition, recent studies have shown that elevated fibrinogen is involved in the malignant behavior of many types of cancer (41-43) and it promotes cancer cell growth, invasion, and metastasis (16-18). Relevant studies have also shown that elevated fibrinogen is associated with tumor progression and metastasis in advanced gastric cancer (19-21). Kijima et al. (44) found that fibrinogen not only plays an important role in the occurrence and metastasis of tumors but can also be used as an observation indicator for the prognosis of esophageal squamous cell carcinoma. Suzuki et al. (45) demonstrated that preoperative plasma fibrinogen elevation was associated with tumor progression and poor overall survival in patients with GC. In addition, Palumbo et al. (46) indicated that in animal experiments fibrinogen deficiency can significantly reduce the potential of tumor metastasis. The current study found that the FLR of the GC with PD group was higher than that of the without

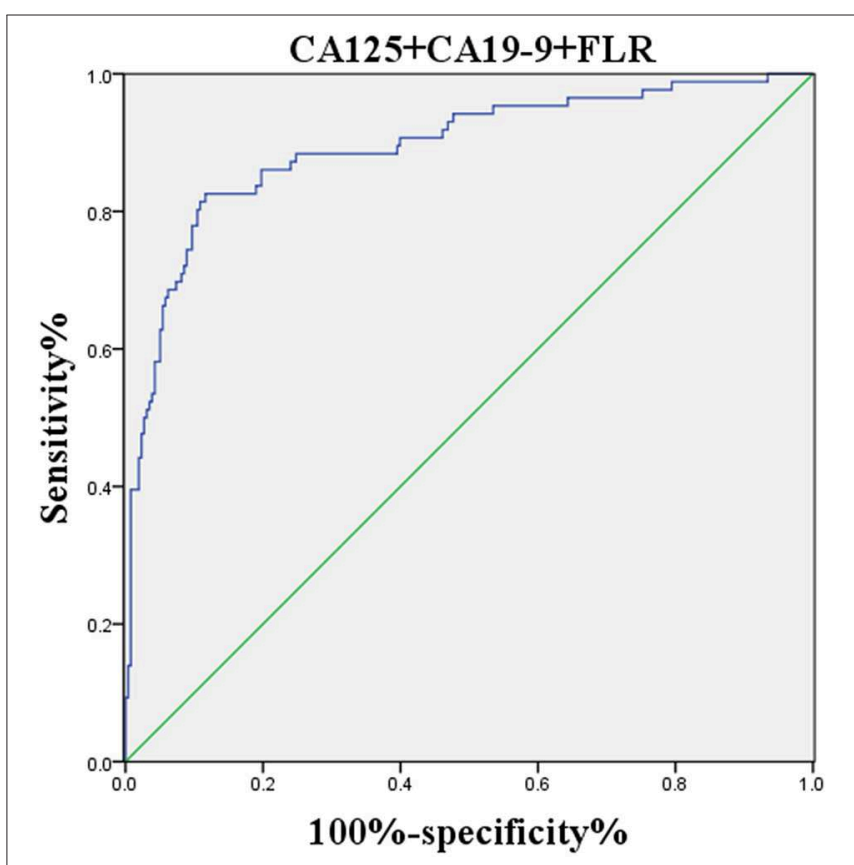

FIGURE 6 | ROC curve of the combined factors.

PD group and the FLR was an independent risk factor for PD in GC. In addition, FLR was also selected in the model of the classification tree. Meanwhile, when the random forest algorithm was applied to extract the important risk factors for PD of GC, these results also showed that FLR was an important risk factor.

CA125 is a tumor-associated glycoprotein antigen and an increase in its serum level is associated with many malignant tumors, and an increased level of CA125 in peritoneal lavage is associated with PD and a poor prognosis of GC (47). Nakata et al. (48) first analyzed the clinical data of 384 patients with GC 
and concluded that the accuracy, sensitivity, and specificity of serum CA125 in the diagnosis of PD of GC were 90.8, 39.4, and 95.7\%, respectively. Hwang et al. (49) showed that the accuracy, sensitivity and specificity of serum CA125 in the diagnosis of PD in GC were 91.5, 38.6, and 98.4\%, respectively. Emoto et al. (8) indicated that serum CA125 was significantly positively correlated with GC with PD, and its diagnostic sensitivity was $46 \%$. In addition, Fujimura et al. (10) found that the accuracy, sensitivity, and specificity of serum CA125 in the diagnosis of PD of GC were 76,55 , and $100 \%$, respectively (14). This study found that serum CA125 of the GC with PD group was higher than that of the without PD group and serum CA125 was an independent risk factor for PD in GC. The accuracy, sensitivity, and specificity of serum CA125 in the diagnosis of PD in GC were $82,79.1$, and $84.9 \%$, respectively. In addition, serum CA125 was the most important determining factor in the model of the classification tree. Meanwhile, when the random forest algorithm was applied to extract the important risk factors of GC with $\mathrm{PD}$, the results also showed that serum CA125 was the most important risk factor.

CA72-4 is a tumor-associated glycoprotein that can be found in a variety of cancers, and has a high specificity for the diagnosis of GC (50). Ca19-9 is a kind of glycoprotein that exists in the form of mucin in serum. Its level in vivo is affected by the size, metastasis, and invasion of tumors, and it is mainly used for the diagnosis of pancreatic cancer, GC, and colon cancer (51). Shimada et al. (52) found through systematic review that serum CA72-4 and CA19-9 were of great value in monitoring the recurrence and metastasis of patients with GC. Emoto et al. (8) showed that the sensitivity of serum CA72-4 and CA19-9 in the diagnosis of GC with PD were 36 and 45\%, respectively, and that serum CA72-4 was a useful indicator of prognosis. Hamazoe et al. (53) demonstrated that the positive rate of serum CA72-4 in patients with PD of GC was significantly higher than that of CEA. Ucar et al. (54) reported that serum levels of CA72-4 and CA19-9 were significantly increased in patients with PD of GC; high preoperative serum CA72-4 levels in patients with GC were associated with a higher risk of death. In addition, Hackbarth et al. (9) indicated that GC with PD can cause peritoneal inflammation, leading to a significant increase in CA19-9 levels, so CA19-9 can be considered as an indicator for the diagnosis of PD in GC. This study found that serum CA72-4 and CA199 of the GC with PD group was higher than that of the without PD group and serum CA19-9 was an independent risk factor for GC with PD. The accuracies of serum CA72-4 and CA19-9 in the diagnosis of PD in GC were 71.7 and $68.4 \%$, respectively; the sensitivities were 57 and 57\%, respectively; and the specificities were 86.4 and $79.8 \%$, respectively. In addition, serum CA72-4 is the second most important determining factor in the model of the classification tree. Meanwhile, when the random forest algorithm was applied to extract the important risk factors of GC with PD, those results also showed that serum CA2-4 and CA19-9 were both relatively important risk factors.

In addition, the decision rules involved four variables (CA125, CA19-9, CA72-4, and FLR) selected by the classification tree program. The accuracy, sensitivity, specificity, positive predictive value and negative predictive value of the decision rules for detecting PD of GC were 89.5, 77.4, 94.0, 82.8, and $91.8 \%$, respectively. Meanwhile, we combined serum CA125, CA19-9, CA72-4, and FLR to construct a nomogram model for risk assessment of GC with $\mathrm{PD}$, and showed that the accuracy, sensitivity and specificity were 91, 89.5, and $79.5 \%$, respectively.

This study has some limitations. First, this is a singlecenter retrospective study, so there may be some bias. Second, the sample size is not sufficiently large. Third, several other inflammatory markers related to tumor progression, such as interleukin-6, C-reactive protein, and hypersensitive Creactive protein, were not included in this study. Therefore, multicenter large-scale prospective randomized controlled trials are necessary.

\section{CONCLUSION}

This is the first study to apply FLR to predict PD of GC and to establish decision rules and risk assessment models for PD of GC. We found that CA125 > 17.3 U/ml, CA19-9 > $27.315 \mathrm{U} / \mathrm{ml}$, and FLR $>2.555$ were risk factors for GC with PD; the decision rules and nomogram models constructed by CA125, CA19-9, CA72-4, and FLR can correctly predict the risk of GC with $\mathrm{PD}$, providing certain guidance for preoperative diagnosis of PD in GC.

\section{DATA AVAILABILITY STATEMENT}

The datasets generated for this study are available on request to the corresponding author.

\section{ETHICS STATEMENT}

All procedures were performed in accordance with the guidelines of the Helsinki Declaration. The study was approved by the Ethical Committee of the Second Affiliated Hospital of Nanchang University and written informed consent was obtained from all patients.

\section{AUTHOR CONTRIBUTIONS}

$\mathrm{CH}$ and $\mathrm{ZZh}$ designed the study and wrote the manuscript with contribution from all authors. CH, ZL, LX, and YX collected clinical data. $\mathrm{CH}$ and $\mathrm{HL}$ analyzed the data. ZZo and JH provided critical comments for this paper. All authors read and approved the final version of the paper.

\section{FUNDING}

This present study was supported by the National Natural Science Foundation of China (Grant Nos. 81560389, 81860433), Key Research and Development Program of Jiangxi Province (Grant No. 20181BBG70015), and Natural Science Youth Foundation of Jiangxi Province (Grant No. 20192BAB215036). 


\section{REFERENCES}

1. Bray F, Ferlay J, Soerjomataram I, Siegel RL, Torre LA, Jemal A. Global cancer statistics 2018: GLOBOCAN estimates of incidence and mortality worldwide for 36 cancers in 185 countries. CA Cancer J Clin. (2018) 68:394424. doi: $10.3322 /$ caac. 21492

2. Maehara Y, Hasuda S, Koga T, Tokunaga E, Kakeji Y, Sugimachi $\mathrm{K}$. Postoperative outcome and sites of recurrence in patients following curative resection of gastric cancer. $\mathrm{Br} J$ Surg. (2000) 87:353-7. doi: 10.1046/j.1365-2168.2000.01358.x

3. Ajani JA, D’Amico TA, Almhanna K, Bentrem DJ, Chao J, Das P, et al. Gastric cancer, version 3.2016, NCCN clinical practice guidelines in oncology. J Natl Compr Cancer Netw. (2016) 14:1286-312. doi: 10.6004/jnccn.2016.0137

4. Association JGC. Japanese gastric cancer treatment guidelines 2014 (ver. 4). Gastric Cancer. (2017) 20:1-19. doi: 10.1007/s10120-016-0622-4

5. Kim SJ, Kim H-H, Kim YH, Hwang SH, Lee HS, Park DJ, et al. Peritoneal metastasis: detection with 16-or 64-detector row CT in patients undergoing surgery for gastric cancer. Radiology. (2009) 253:40715. doi: $10.1148 /$ radiol.2532082272

6. Yang Q-M, Bando E, Kawamura T, Tsukiyama G, Nemoto M, Yonemura $\mathrm{Y}$, et al. The diagnostic value of PET-CT for peritoneal dissemination of abdominal malignancies. Gan To Kagaku Ryoho. (2006) 33:1817-21.

7. Koh J-L, Yan TD, Glenn D, Morris DL. Evaluation of preoperative computed tomography in estimating peritoneal cancer index in colorectal peritoneal carcinomatosis. Ann Surg Oncol. (2009) 16:327-33. doi: 10.1245/s10434-008-0234-2

8. Emoto S, Ishigami H, Yamashita H, Yamaguchi H, Kaisaki S, Kitayama J. Clinical significance of CA125 and CA72-4 in gastric cancer with peritoneal dissemination. Gastric Cancer. (2012) 15:154-61. doi: 10.1007/s10120-011-0091-8

9. Hackbarth JS, Murata K, Reilly WM, Algeciras-Schimnich A. Performance of CEA and CA19-9 in identifying pleural effusions caused by specific malignancies. Clin Biochem. (2010) 43:1051-5. doi: 10.1016/j.clinbiochem.2010.05.016

10. Fujimura T, Kinami S, Ninomiya I, Kitagawa H, Fushida S, Nishimura G, et al. Diagnostic laparoscopy, serum CA125, and peritoneal metastasis in gastric cancer. Endoscopy. (2002) 34:569-74. doi: 10.1055/s-2002-33228

11. Balkwill F, Mantovani A. Inflammation and cancer: back to Virchow? Lancet. (2001) 357:539-45. doi: 10.1016/S0140-6736(00)04046-0

12. Mantovani A, Allavena P, Sica A, Balkwill F. Cancer-related inflammation. Nature. (2008) 454:436. doi: 10.1038/nature07205

13. Tennent GA, Brennan SO, Stangou AJ, O'Grady J, Hawkins PN, Pepys MB. Human plasma fibrinogen is synthesized in the liver. Blood. (2007) 109:19714. doi: 10.1182/blood-2006-08-040956

14. Perisanidis C, Psyrri A, Cohen E, Engelmann J, Heinze G, Perisanidis B, et al. Prognostic role of pretreatment plasma fibrinogen in patients with solid tumors: a systematic review and meta-analysis. Cancer Treat Rev. (2015) 41:960-70. doi: 10.1016/j.ctrv.2015.10.002

15. Tang L, Liu K, Wang J, Wang C, Zhao P, Liu J. High preoperative plasma fibrinogen levels are associated with distant metastases and impaired prognosis after curative resection in patients with colorectal cancer. J Surg Oncol. (2010) 102:428-32. doi: 10.1002/jso.21668

16. Jiang H-G, Li J, Shi S-B, Chen P, Ge L-P, Jiang Q, et al. Value of fibrinogen and D-dimer in predicting recurrence and metastasis after radical surgery for non-small cell lung cancer. Med Oncol. (2014) 31:22. doi: 10.1007/s12032-014-0022-8

17. Matsuda S, Takeuchi H, Kawakubo H, Fukuda K, Nakamura R, Takahashi $\mathrm{T}$, et al. Cumulative prognostic scores based on plasma fibrinogen and serum albumin levels in esophageal cancer patients treated with transthoracic esophagectomy: comparison with the Glasgow prognostic score. Ann Surg Oncol. (2015) 22:302-10. doi: 10.1245/s10434-014-3857-5

18. Sheng L, Luo M, Sun X, Lin N, Mao W, Su D. Serum fibrinogen is an independent prognostic factor in operable nonsmall cell lung cancer. Int $J$ Cancer. (2013) 133:2720-5. doi: 10.1002/ijc.28284

19. Lee SE, Lee JH, Ryu KW, Nam B-H, Cho SJ, Lee JY, et al. Preoperative plasma fibrinogen level is a useful predictor of adjacent organ involvement in patients with advanced gastric cancer. J Gastric Cancer. (2012) 12:817. doi: $10.5230 /$ jgc.2012.12.2.81
20. Yamashita H, Kitayama J, Nagawa H. Hyperfibrinogenemia is a useful predictor for lymphatic metastasis in human gastric cancer. Jpn J Clin Oncol. (2005) 35:595-600. doi: 10.1093/jjco/hyi150

21. Yamashita H, Kitayama J, Kanno N, Yatomi Y, Nagawa H. Hyperfibrinogenemia is associated with lymphatic as well as hematogenous metastasis and worse clinical outcome in T2 gastric cancer. BMC Cancer. (2006) 6:147. doi: 10.1186/1471-2407-6-147

22. Türe M, Kurt I, Kürüm T. Analysis of intervariable relationships between major risk factors in the development of coronary artery disease: a classification tree approach. Anatol J Cardiol/Anadolu Kardiyol Derg. (2007) 7:140-5.

23. Heikes KE, Eddy DM, Arondekar B, Schlessinger L. Diabetes Risk Calculator: a simple tool for detecting undiagnosed diabetes and pre-diabetes. Diabetes Care. (2008) 31:1040-5. doi: 10.2337/dc07-1150

24. Xin Z, Yuan J, Hua L, Ma Y-H, Zhao L, Lu Y, et al. A simple tool detected diabetes and prediabetes in rural Chinese. J Clin Epidemiol. (2010) 63:10305. doi: 10.1016/j.jclinepi.2009.11.012

25. Breiman L. Random forests. Mach Learn. (2001) 45:532. doi: 10.1023/A:1010933404324

26. Wang Y, Li J, Xia Y, Gong R, Wang K, Yan Z, et al. Prognostic nomogram for intrahepatic cholangiocarcinoma after partial hepatectomy. J Clin Oncol. (2013) 31:1188-95. doi: 10.1200/JCO.2012.41.5984

27. Kayaalp C, Arda K, Orug T, Ozcay N. Value of computed tomography in addition to ultrasound for preoperative staging of gastric cancer. Eur J Surg Oncol. (2002) 28:540-3. doi: 10.1053/ejso.2002.1296

28. Yajima K, Kanda T, Ohashi M, Wakai T, Nakagawa S, Sasamoto R, et al. Clinical and diagnostic significance of preoperative computed tomography findings of ascites in patients with advanced gastric cancer. Am J Surg. (2006) 192:185-90. doi: 10.1016/j.amjsurg.2006.05.007

29. Bando E, Yonemura Y, Taniguchi K, Yasui T, Fushida S, Fujimura $\mathrm{T}$, et al. Intraoperative lavage for cytological examination in 1,297 patients with gastric carcinoma. Am J Surg. (1999) 178:256-62. doi: 10.1016/S0002-9610(99)00162-2

30. Benevolo M, Mottolese M, Cosimelli M, Tedesco M, Giannarelli D, Vasselli S, et al. Diagnostic and prognostic value of peritoneal immunocytology in gastric cancer. J Clin Oncol. (1998) 16:3406-11. doi: 10.1200/JCO.1998.16.10.3406

31. Coussens LM, Werb Z. Inflammation and cancer. Nature. (2002) 420:8607. doi: $10.1038 /$ nature 01322

32. Coussens LM, Zitvogel L, Palucka AK. Neutralizing tumorpromoting chronic inflammation: a magic bullet? Science. (2013) 339:286-91. doi: 10.1126/science. 1232227

33. Quigley DA, Kristensen V. Predicting prognosis and therapeutic response from interactions between lymphocytes and tumor cells. Mol Oncol. (2015) 9:2054-62. doi: 10.1016/j.molonc.2015.10.003

34. Eo WK, Da Wun Jeong HJC, Won KY, Choi SI, Kim SH, Chun SW, et al. Absolute monocyte and lymphocyte count prognostic score for patients with gastric cancer. World J Gastroenterol. (2015) 21:2668. doi: 10.3748/wjg.v21.i9.2668

35. Arigami T, Uenosono Y, Ishigami S, Matsushita D, Hirahara T, Yanagita $\mathrm{S}$, et al. Decreased density of $\mathrm{CD} 3+$ tumor-infiltrating lymphocytes during gastric cancer progression. J Gastroenterol Hepatol. (2014) 29:143541. doi: $10.1111 /$ jgh. 12551

36. Zacharski LR, Meehan KR, Algarra SM, Calvo FA. Clinical trials with anticoagulant and antiplatelet therapies. Cancer Metastasis Rev. (1992) 11:421-31. doi: 10.1007/BF01307191

37. Palumbo JS, Talmage KE, Massari JV, La Jeunesse CM, Flick MJ, Kombrinck $\mathrm{KW}$, et al. Platelets and fibrin (ogen) increase metastatic potential by impeding natural killer cell-mediated elimination of tumor cells. Blood. (2005) 105:17885. doi: 10.1182/blood-2004-06-2272

38. Ridker PM, Howard CP, Walter V, Everett B, Libby P, Hensen J, et al. Effects of interleukin-1 $\beta$ inhibition with canakinumab on hemoglobin Alc, lipids, C-reactive protein, interleukin-6, and fibrinogen: a phase IIb randomized, placebo-controlled trial. Circulation. (2012) 126:273948. doi: 10.1161/CIRCULATIONAHA.112.122556

39. Zheng S, Shen J, Jiao Y, Liu Y, Zhang C, Wei M, et al. Platelets and fibrinogen facilitate each other in protecting tumor cells from natural killer cytotoxicity. Cancer Sci. (2009) 100:859-65. doi: 10.1111/j.1349-7006.2009. 01115.x 
40. Goicoechea M, De Vinuesa SG, Gómez-Campderá F, Aragoncillo I, Verdalles $\mathrm{U}$, Mosse A, et al. Serum fibrinogen levels are an independent predictor of mortality in patients with chronic kidney disease (CKD) stages 3 and 4: new strategies to prevent cardiovascular risk in chronic kidney disease. Kidney Int. (2008) 74:S67-70. doi: 10.1038/ki.2008.519

41. Qi Q, Geng Y, Sun M, Chen H, Wang P, Chen Z. Hyperfibrinogen is associated with the systemic inflammatory response and predicts poor prognosis in advanced pancreatic cancer. Pancreas. (2015) 44:97782. doi: 10.1097/MPA.0000000000000353

42. Liu Y-L, Lu Q, Liang J-W, Xia Y, Zhang W, Hu B-Q, et al. High plasma fibrinogen is correlated with poor response to trastuzumab treatment in HER2 positive breast cancer. Medicine. (2015) 94:e481. doi: 10.1097/MD.0000000000000481

43. Thurner E-M, Krenn-Pilko S, Langsenlehner U, Stojakovic T, Pichler $\mathrm{M}$, Gerger A, et al. The association of an elevated plasma fibrinogen level with cancer-specific and overall survival in prostate cancer patients. World J Urol. (2015) 33:1467-73. doi: 10.1007/s00345-0141459-2

44. Kijima T, Arigami T, Uchikado Y, Uenosono Y, Kita Y, Owaki T, et al. Combined fibrinogen and neutrophil-lymphocyte ratio as a prognostic marker of advanced esophageal squamous cell carcinoma. Cancer Sci. (2017) 108:193-9. doi: 10.1111/cas.13127

45. Suzuki T, Shimada H, Nanami T, Oshima Y, Yajima S, Ito M, et al. Hyperfibrinogenemia is associated with inflammatory mediators and poor prognosis in patients with gastric cancer. Surg Today. (2016) 46:1394401. doi: 10.1007/s00595-016-1339-z

46. Palumbo JS, Potter JM, Kaplan LS, Talmage K, Jackson DG, Degen JL. Spontaneous hematogenous and lymphatic metastasis, but not primary tumor growth or angiogenesis, is diminished in fibrinogen-deficient mice. Cancer Res. (2002) 62:6966-72.

47. Yamamoto M, Baba H, Toh Y, Okamura T, Maehara Y. Peritoneal lavage CEA/CA125 is a prognostic factor for gastric cancer patients. J Cancer Res Clin Oncol. (2007) 133:471-6. doi: 10.1007/s00432-0060189-2

48. Nakata B, Chung KHY, Kato Y, Yamashita Y, Maeda K, Onoda N, et al. Serum CA 125 level as a predictor of peritoneal dissemination in patients with gastric carcinoma. Cancer. (1998) 83:2488-92. doi: 10.1002/(SICI)10970142(19981215)83:12<2488::AID-CNCR12>3.0.CO;2-1

49. Hwang GI, Yoo CH, Sohn BH, Shin JH, Park YL, Dai Kim H, et al. Predictive value of preoperative serum CEA, CA19-9 and CA125 levels for peritoneal metastasis in patients with gastric carcinoma. Cancer Res Treat. (2004) 36:178. doi: 10.4143/crt.2004.36.3.178

50. Kim DH, Oh SJ, Oh CA, Choi MG, Noh JH, Sohn TS, et al. The relationships between perioperative CEA, CA 19-9, and CA 72-4 and recurrence in gastric cancer patients after curative radical gastrectomy. J Surg Oncol. (2011) 104:585-91. doi: 10.1002/jso.21919

51. Kim J-H, Jun K-H, Jung H, Park I-S, Chin H-M. Prognostic value of preoperative serum levels of five tumor markers (Carcinoembryonic Antigen, CA19-9, Alpha-fetoprotein, CA72-4, and CA125) in gastric cancer. Hepato Gastroenterol. (2014) 61:863-9.

52. Shimada H, Noie T, Ohashi M, Oba K, Takahashi Y. Clinical significance of serum tumor markers for gastric cancer: a systematic review of literature by the Task Force of the Japanese Gastric Cancer Association. Gastric Cancer. (2014) 17:26-33. doi: 10.1007/s10120-013-0259-5

53. Hamazoe R, Maeta M, Matsui T, Shibata S, Shiota S, Kaibara N. CA72-4 compared with carcinoembryonic antigen as a tumour marker for gastric cancer. Eur J Cancer. (1992) 28:1351-4. doi: 10.1016/0959-8049(92)90517-6

54. Ucar E, Semerci E, Ustun H, Yetim T, Huzmeli C, Gullu M. Prognostic value of preoperative CEA, CA 19-9, CA 72-4, and AFP levels in gastric cancer. Adv Ther. (2008) 25:1075-84. doi: 10.1007/s12325-008-0100-4

Conflict of Interest: The authors declare that the research was conducted in the absence of any commercial or financial relationships that could be construed as a potential conflict of interest.

Copyright (c) 2019 Huang, Liu, Xiao, Xia, Huang, Luo, Zong and Zhu. This is an open-access article distributed under the terms of the Creative Commons Attribution License (CC BY). The use, distribution or reproduction in other forums is permitted, provided the original author(s) and the copyright owner(s) are credited and that the original publication in this journal is cited, in accordance with accepted academic practice. No use, distribution or reproduction is permitted which does not comply with these terms. 